\title{
Study on Characteristics of Mobile Network Information Dissemination in Universities
}

\author{
Cui Jiangning \\ Shandong Women's University, Shandong Jinan, 250300 \\ cuijiangning@sdwu.edu.cn
}

\begin{abstract}
The phone network has acted as a part of learning and life for contemporary students. Rich resources and a wide range of information on the network, which has effected profoundly on college students' habits and learning styles. Based on the analysis and research of propagation characteristics by mobile phone network in universities, it is proved that mobile networks have played an important role on fitness of college students in group, the attitudes of participating in information dissemination, applying information dissemination to promote the core, meeting their needs of internal and external communication included. While its negative impacts cannot be ignored, it should be confronted actively and guided timely. Take advantage of the phone network information dissemination to develop talent training roundly and then improve the comprehensive quality of high-level personnel constantly.
\end{abstract}

Keywords: university; mobile phone network; information propagation; characteristics

With the rapid development of the information society, science and technology, the network has become one of the main channels for the transmission of information in today's society, to some extent; it is the main channel. The mobile network owns the highest utilization rate of all. According to the latest statistics from the China Internet Network Information Center (CNNIC) which released in January 2014 showed that mobile phone Internet is still the main power of netizens scale growth: As of December 2013, Chinese netizens up to 618 million, a total of 53.58 million Internet users were created throughout the year. Internet penetration was $45.8 \%$, up of 3.7 percentage points compared with the end of 2012. Chinese mobile phone users reached 500 million, increased 8,009 people by the end of 2012, Internet users in the use of mobile Internet population accounted for $81 \%$. The proportion of Chinese Internet users access the Internet via laptops and desktop computers were $69.7 \%$ and $44.1 \%$ respectively, compared to an annual average decline in 2012 , a decrease of the ratio was 0.8 percentage points and 1.8 percentage points respectively. The proportion of mobile Internet maintained a rapid growth, up from $74.5 \%$ to $81.0 \%$, improved 6.5 percentage points, annual growth rate of $19.1 \%$, continuing to maintain its status as the largest Internet terminal. The proportion of new Internet users becoming higher and higher proved that mobile phone also plays a catalytic role in the increasing rate of Internet users. Among the proportion of new Internet users, the mobile Internet users in China added up to 73.3\%, much higher than the proportion of the Internet users via other devices, thus the mobile phone is still the main driving force for the growth of Internet users in China. The college students represent the highest proportion of all mobile phone users, the data of previous surveys since 1997 by China Internet Network Information Center showed that teenagers under 24 years old has always been China's network users' main group. During the process of rapid development of mobile networks, highly adventurous and innovative spirit of the young university student groups once again becoming a dominant mobile phone neticizen group once again. 
Mobile Internet refers to link to the Internet by use of the mobile phone via WAP protocol supported Web browsers, so as to achieve the purpose of browsing the Web. According to the author observed at ordinary times, the current college students each has a mobile phone which access internet basically. With mobile Internet, students can browse the Web, access information, online shopping, but also through QQ, microblogging, letters, SNS and other network platform, to transmit information, express feelings, discuss issues, virtual social networking and many other features, based on college students age and psychological characteristics, it can be said in college today's society, the mobile network has become a university student learning, life indispensable medium. Therefore, Study on characteristics of mobile network information dissemination in universities is benefited for us comprehending contemporary college students 'ideological dynamics and their motivation of behavior, as well as carrying out targeted education training to cultivate high level talents which adapted to diversified and rapid social development society.

Unlike one-way information dissemination model of traditional media (radio, television, newspapers, magazines), the mobile phone network with a powerful interactive technology to create a new media landscape, promoted the Internet's participation and interactivity to a higher stage .The user is no longer a passive acceptance in the process of dissemination of information, but active information creators, communicators and recipients. Meanwhile, because of college students easy to accept new things, have a certain scientific knowledge, emotional, dare to express, have no pressure for further studies or work, own more free time, but the outlook on life values is not stable, contact with media literacy is not deep enough. Therefore, the universities phones network information dissemination is always tend to exhibit different characteristics with other groups.

\section{The Characteristic of University Student Population Turns out to be Much More Adaptable to the Mobile Phone Network Information Dissemination Platform than Other Groups}

As a special group, university student population has its own characteristics different from other groups. This feature gives them advantages in adapting the form of spread information by the phone network. As college students in school, learning is still their main task, they are different from junior or senior students, they have autonomous learning desire and ability, smiles classroom knowledge cannot meet their needs, for them, a variety of expertise and knowledge is what they really pursue. Of all the methods, the mobile phone network is the most convenient one. According to the surveys, "the expansion of knowledge" has become one of the main purposes of college students using a mobile phone network, while socialize by phone network become another important function for them. This kind of mobile network terminal product have become an indispensable tool for their learning and life.

Unlike point-and-shoot cameras, not anyone can use the mobile phone network which is based on the powerful electronic interactive technology; it is a kind of information dissemination media with high-tech. Only when the uses have high quality, can you give full play to the function of the medium. It requires not only the basic hardware configuration, which is a mobile phone with the capable of supporting web browser, but also a certain software configuration, which is able to have a basic knowledge of the mobile Internet; A strong thirst for knowledge, found solutions by consulting others or query network for the unknown; have much free time and disposable funds, dare to try new things on the network, such as mobile phone online shopping; have excellent ability to adapt, you can rapidly operate new things, such as micro message, easy message and other social networks after QQ and microblogging, Just for college students with the above conditions and qualities. In comparison, although young and energetic, because of 
pressure of accessing to academic and a lack of dominant funds, Internet interpersonal interaction is not their main life for high school students. The young people stepped into the workplace, although have their own income, as to the pressures come from work and family lead them lack of time and energy which make them far away from the mobile phone network virtual networking. The main functions of the mobile phone network is also limited to meet the needs of basically communicate information, which is the basically target of Internet. More importantly, college students has a strong spontaneously organized such as some interest community, the association and so on, this spontaneously organized is very similar to what mobile network information dissemination has, which enabling the network information transmission be widely used among college students. At the same time, college students are well educated, focus on social hot problem and can think and action actively. In May 2014, mobile phone network has played a very important role between the spread process of 11 xinjiang uygur college students' open letter to criticize the Violence could case. They discuss and determine the action among the micro letter firstly, and has carried on wide spread through computer, Twitter on mobile phone, which has caused great repercussions.

College students personality have distinct individuality, active thought and diversified values, but they are often self-contradictory, it become virtualization in their interpersonal communication and access to information. Based on these features, except as a basis tools for communication and information query, Mobile network as a new interpersonal platform has become effective means of exchanging information, showing themselves, even gathering opinions, finding contradictions, timely guidance for the college students' self-education.

\section{College Students Actively Participate in the Dissemination of Information through Mobile Phone Network, Chat, Information, and Micro-message has become the Three Main Functions of Information Dissemination}

The convenience of the mobile phone network is unmatched by traditional networks, and college students is a particularly active group in the dissemination of information, we can see students surfing the internet here and there on campus. The information dissemination by mobile network has the features of high speed, wide coverage, low-cost, easy to carry, not limited by time and space. The social-based comprehensive platform, not only has greater communication capabilities, but also increased social communication categories such as information-sharing applications. College students achieve a direct and efficient interaction with each other, timely and rapidly get the information, as well as express their views through QQ, microblogging, micro message, SNS and other social networking platforms. Mobile network is becoming the dominant platform for information dissemination in universities because of its unique media characteristic and media attributes. Currently, the mobile phone network as a medium has been widely used to information dissemination in the university campus, it disseminates information not only high speed, but also large capacity, a variety of information, especially some important information or the agenda-setting information disseminate in large-scale and high speed as virus fission. The attention of a message by thousands of people is not a rare phenomenon any longer; its efficiency is much higher than in the realistic society disseminated by individuals and organizations, and even the mass media. Such as the concern of MH370 flight incident, and the president xi buy steamed buns in qingfeng, reflects the university's mobile network disseminate information actively.

According to a survey of network usage in Changzhou universities showed that the rate of mobile Internet usage reached $93.7 \%$. There are almost $60 \%$ of people think the mobile Internet is more convenient than a computer. The survey showed that frequently used project in the mobile Internet, the basic interpersonal chat function area, reached $85 \%$ of 
investigation number, followed by browsing information and news, accounting for $65 \%$.It can be said that mobile network has become a major channel for college students to access information. Currently, there are many colleges and universities have already passed information which closely linked to their life and learning through mobile phone network to the students such as query results, curriculum inquiry, inquiry classroom situations, seminars inquiries and so on. In addition, In addition, the presence of the micro-message will promote network relationships and individual information dissemination to another climax. Based on the origin of "information system", the micro message has not only let in two separate places people can talk to each other, video chat, share information, real-time intercom communication tool, it is building up a huge "I / O platform to attract student users in by sharing mechanism, launch circle of friends and the public platform and start up the multiple functions of shake, sweep in which make friends in long-distance search and locate etc. Micro-message eco-system are the embodiment of respect for the wishes of the user from the beginning to the next period of time, Take pictures show as the core, with a brief textual representation of information dissemination, making the micro-channel accepted by more students. The university makes the original blunt direct message notifications into illustrations, interesting and understandable through micro message. In this way, many of the traditional didactic education, particularly the ideological and political education, updated with developing society, closed to the characteristics of students in this era, information dissemination through the mobile phone network has become acceptable to students. Currently, chat, information, and micro-message has become the three major functions of information dissemination by mobile phone network. According to another survey of surfing the Internet with cell phone in Henan universities showed that there is $28.9 \%$ students spend less than 1 hour on internet, $42.4 \%$ between 1 to 3 hours, $19.8 \%$ between 3 to 5 hours and 9.8\% more than 5 hours. We can see that college students spend a long time in internet by cell phone every day. College students' cell phone use most often do is to search online browsing information, news, accounting for $47.6 \%$ of the total. Followed by the Internet chatting, accounting for $27 \%$ of the total.

During the various mobile phone network system, micro-letter as a mobile instant messaging software which supports for multiple platforms and aims to promote the exchange of human communication, are increasingly popular between people, especially college students . A study about college students' using condition of micro-letter on internet shows that 54\% college students are using micro-letter, and $96 \%$ students are using, understanding or interested in it; students spend 0-6 hours on line every day accounted for $58 \%$; $42 \%$ of students are willing to tell their feelings with a stranger in micro-letter. This means that micro-letter has integrated into the life of college students in a considerable extent. The emergence of micro-letter will develop network relationships and individual information transmitted to another climax. Based on the origin of "information system", micro-letter is not only a tool which can make separated people talk to each other, video chat, share information, real-time intercom communication, but also building a huge "platform of I/O". Micro-letter have a lot of features, such as many functions, low flow consumption, suitable for most smart phones, and support multiple languages and data network, it reduced the college students of communication cost in most cases, for their pursuit of fashion, strong applicability and low cost, it is indeed a very good software. 

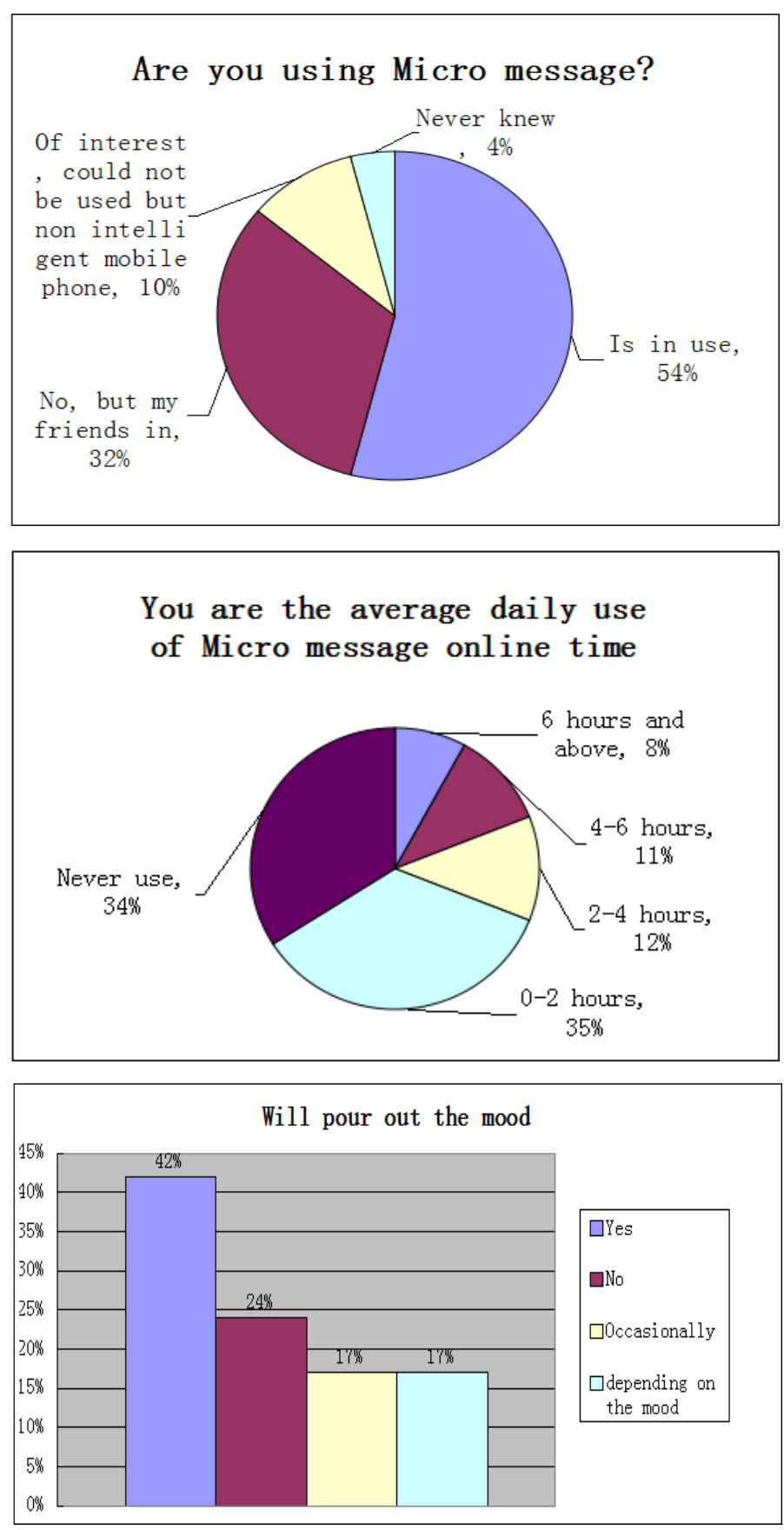

As educators in colleges and universities, also can give full play to this advantage of micro-letter,they can change the original curt information notification directly into illustrated, interesting and easy to understand communication at a relatively low cost, and make many traditional moralistic education, especially ideological education, to keep pace with The Times, close to the age characteristics of students, through the mobile phone network information transmission, it become easily accepted by college students, so as to improve the effect of education .So in the moment, chat, information, and micro letter has become the three functions of mobile network information transmission. 


\section{As Opinion Leaders, Some Students becoming the Core of Promoting through the Mobile Phone Network for Promoting Information Dissemination in the University}

At the perspective of communication, the students group has its own unique characteristics in the dissemination of information, they have not yet step into society, is relatively purity; The outlook on life values still immature, easily to be extremely; relatively simple access to information channels, the information is correct judgment is not accurate; for the dissemination of information the college students group is always dominated by the emotions and generated blindly behavior. Universities mobile network information dissemination showing strong self-organized and authenticity, which can say to be another form of continuation and expansion of intersection in the real society and groups through the mobile network. In the real world, people stick a label to others and belongings identified according to blood relationship, organization relationship, the geographical position. The mobile network in university link students with common background or interests. The features of dispersion and concealment in traditional network have decreased in the mobile network. In this case, some students with independent-minded in usual daily life, deeply thought, well informed, and a certain cachet, will take this advantage to the mobile network to continue becoming the opinion leaders in the mobile network information dissemination. These people are often the first point of contacting information from the school outside, without these core groups to participate in the information dissemination, the information is difficult to spread in the colleges and universities. Meanwhile, those who spread the information within the university also played a role of central driving power. They are generally actively worked on a few important plates in mobile networks. They also have the ability of remarking about spontaneous organized opinions through mobile network. For information dissemination, for information dissemination, these small groups of opinion leaders occupied the vast majority of information dissemination. The number of these opinion leaders, though less than $10 \%$, spread their personal willingness and ability to generate a significant impact on the spread of network information.

We all know that the mobile phone network dissemination of information is often presented as the spread of viral or explosive. Because of the mobile network in the universities having the feature of organized spontaneous, forming various circles which are similar with circle of friends, the self-organize circle is based on people who know each other. People are always trust in information dissemination between acquaintances, which results in information disseminates as fast as the fission of virus in a specific group. This rapid spread is precisely involved and derived by these opinion leaders to attend. These opinion leaders actually represent a user circle, the information nested and connected in the circle of different users to carry out a wide range of transmission. This feature also puts forward higher requirements on these opinion leaders, and any comments from them should be practical and realistic, objective and fair, accord with thinking habit and psychological characteristics of college students, it is not easy for the students, they are fullhearted, pursuiting truth,but often confused by the superficial phenomenon. Su Qiong and Lai Guowei from Xiamen university proposed: in the aspect of gathering information, college students' preference gap is little in perception and intuition. And in the evaluation of information, more than $70 \%$ of the students towards emotional cognitive style.So we learn that when college students assessment information lay particular stress on emotion, concern he harmonious relationship between themselves with others, like to use subjective judgment words in communication in order to enhance their ability of persuade and judge. Therefore, as educators in colleges and universities, should pay close attention to these opinion leaders, remind them to know the truth behind the events in time, guides them to look at the problems dialectically, and even become a opinion leader who is accepted by college students, to create another position in 
ideological education of higher learning. Such as during the "9.18" national calamity after diaoyu islands event, educators should guide students' patriotic enthusiasm actively, neither can hit their patriotic enthusiasm, but to make their emotional catharsis properly. In this case, virtual college students mainly gather groups using SMS, QQ, Twitter, micro letter,via the mobile phone network platform, spread correct mainstream speech widely, so that the students can realize the event more fully, then to make a rational judgment.

\section{Meet the Needs of Student Groups' Psychological Demands, Expanding their Daily Interaction Patterns}

College students are not only active thinking, rich in emotion, enthusiastic to make friends, inspired to show themselves, but also has a strong self-esteem, they need effective communication and release channels to get rid of resisted emotions from individual communication face to face. Mobile network information dissemination offers more freedom; give the students a relatively open platform for communication, which completely accords with the physiological and psychological characteristics of college students. It avoids the stress and embarrassment of face-to-face dissemination to some extent; make the information dissemination as multi-directional interaction. Different opinions can be accepted by different people. The barriers of physical media will make communication between two or more parties far away from extremely conducts. What's more, And enabling them to elaborate, sort out their emotion as well as inform the other, through reasonable means to meet their psychological demands, expressing their happy or sad moods, and find a way to solve the detected problems.

Mobile network information dissemination not only meet the demands of college students in the inner psychological needs, but also expand their mode of outward daily interactions as an important channel. College students are eager to interact with the world outside, but in real society, the environment of university is relatively independent, the college students are mainly in contact with schoolmates, teachers and friends, unable to meet their needs of extensive and hierarchy contacts with the outside world. While through the mobile phone network, can extend their scope of interpersonal communication, to extend the time of interpersonal communication, to get more information, truly "a scholar need not leave his home, can know what's going on in the world" Also for college students to enter society in the future accumulate a certain exchanges of experience and network resources.

\section{The Mobile Phone Network has Significantly Negative Impact on the Dissemination of Information, Should be Timely Guidance and Education}

Everything has pros and cons, things with more powerful function, its positive and negative influence complementary to each other more deeply.properly use of mobile network information dissemination, can play its positive energy, enrich the campus life for college students, improve the learning efficiency, and create a good campus culture. However, in the current university, it is very obvious that the negative impact from the mobile phone network dissemination of information .According to investigation, the majority of college most of the college students use mobile phone network there is a "long time usage, involving a narrow field and entertainment-oriented," etc. Although the mobile phone network is the main channel for college students to get information, is an effective complement students' interpersonal communication. If students use the mobile phone internet in wrong way, it has striking consequences on students whose self-control ability is not strong and weak external constraints. Mobile Internet is very convenient, students often occupy a lot of time, leading to delays in learning, disrupting normal daily routine of life, some of the college students' senses are fuzzy, and some college students 
could not get a correct attitude to the internet information, make their judgment from the partial information and believe it without any doubt. Some students caught in the wrong direction of cognitive, even some of them were thrown into the jail because of spread rumors. Psychological research shows that, due to people's natural endowment of outside information security, people has a natural proximity and high attention to the negative information which may affect the information security in outside world. On October 20, 2011, for example, pi **from Chongqing jiaotong university,grade 2006, institute of civil engineering and construction ,Posting in baidu chong talk bar: "feel hot, Pin CI events actually make to chongqing",which caused many netizens attention and reply. Pin CI events information quickly spread among some students in this university, and caused a certain degree of mood instability.In accordance with the law to the public security ,the police detained him 3 days.

Especially during the political and economic events happening at home and abroad, the mobile phone network information transmission efficiency, often make students have intensity of herd behavior, such as the diaoyu islands event. Although most of the students applying mobile networks tend to be more self-emotion expressing and communicating, which resulted in self-centered cognition, and lack of the unity cooperation ability in the real social communication, which is worried by everyone. In November 2011, for example, someone spread a message in the Internet and mobile phone text messages : peoples from Xinjiang Province transmission HIV/AIDS through blooding food, many people infected with HIV. This information spread rapidly in the university students, causing panic for a time.

For this negative impact of higher education shall promptly take measures to educate not only by traditional means, but also the use of modern technology for deep and comprehensive guide. Actively create a good mobile network environment, rather than confrontation with the establishment of public schools phone network platform for information exchange, contact with students in-depth, a comprehensive understanding of the students' ideological trends to carry out ideological and political education. Meanwhile, college students can use this platform to get school-related resources, and to make reasonable suggestions and comments to school. Moreover, you can also implement targeted individualized education, covert network allows students daring to speak their minds, and the teacher can prompt psychological counseling, to solve the problem.

Universities mobile network information dissemination shares the common characteristic in today's society, but also has their own characteristics. College students are a special group, our country focus on training them on high-level personnel in all aspects,as a great asset to the modern information society, in a sense, their comprehensive quality affect China's culture trend of the future society in a great extent. Mobile phone networks information dissemination since have become an important part of their life, we should be research in-depth, analyze its characteristics, understand it in both positive and negative impacts on the new era in the process of training new talents. Only when can exceed and fully play its positive role. This paper is in terms of research methods, such as the research object is still not perfect, the next step is establishing research to strengthen information dissemination model by the mobile phone network, as well as its influence and so on. 


\title{
References
}

[1] X. Yu, "The propagation characteristics of the campus network information and user influence research", $\mathrm{PhD}$ thesis, (2012).

[2] Report China Internet Network Information Center (CNNIC), "The 33rd China Internet Development Statistics", Baidu library.

[3] Z. Hao, C. Fang, "Mobile Internet environment under the status quo campus information needs and trends research", Jianghan University (Natural Science), (2013), pp. 53-56.

[4] Baidu library, "The problem with the analysis of the mobile phone network lives of college students".

[5] Z. Ni and D. Weiting, "Mobile networks and traditional networks and their impact on college students", The Information Research, (2012), pp. 10-12.

[6] H. Yan, "The research of mobile Internet's influences and solutions on college students' interpersonal communication", master's thesis, (2013).

[7] L. Kun, "Introduction to the negative impact of the mobile Internet on college students in colleges and universities- university in Henan province", Baidu library.

[8] Z. Di, "The influence of the network hot issues on college students' information cognitive style", master's thesis, (2013).

[9] J. Hui, "On the Micro message of Contemporary College Students, Inheritance \& Innovation", (2013).

[10] S. Ting, "Application of the information transmission and interaction of computer network technology", Computer CD Software and Applications, (2014).

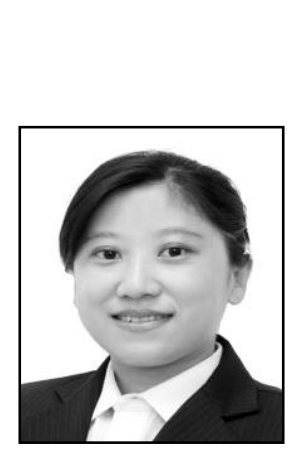

\begin{abstract}
Author
Cui Jiangning, she graduated from Shandong University professional communication, graduate student degree, received master's degree in literature, China in 2007 .She is currently working in a women's college in shandong, associate professor title. She is currently researching on teaching and making scientific survey in communication, advertising, and chaired or participated in several related provincial departmental level subject.
\end{abstract}


International Journal of Multimedia and Ubiquitous Engineering

Vol. 10, No. 3 (2015) 